\title{
Facile Synthesis of High Luminescent Blue Emitting ZnS Quantum Dots for UV to Visible Light Conversion, Solar Cell and LED Applications.
}

\author{
Gerardo Saavedra Rodriguez ${ }^{1}$, F. Javier Carrillo Pesqueira ${ }^{1}$, Raúl Sánchez Zeferino ${ }^{2}$, Roberto C. Carrillo \\ Torres $^{2}$, Álvaro Flores Pacheco ${ }^{1}$, Celso Velásquez O. ${ }^{3}$, Roberto P. Duarte ${ }^{2}$, M. Enrique Alvarez R. ${ }^{2}$ \\ 1. Posgrado en Nanotecnología, Universidad de Sonora, Hermosillo, Sonora, México, C.P. 83000. \\ 2. Departamento de Física, Universidad de Sonora, Hermosillo, Sonora, México, C.P. 83000. \\ 3. Centro de Investigación en Nanociencia y Nanotecnología Universidad de Guadalajara, C.P. 46600, \\ Ameca, Jalisco México.
}

Nanostructured semiconductors have been widely studied since last decade due to the clear and desirable improvement of some of their properties, such as optical, electrical, magnetic, etc. In recent years, the interest to modify those properties by reducing the particle size of systems, until reach the quantum dot (QD) scale, has increased [1,2]. In QD scale, each molecular, even atomic, energy levels influence more significantly over electron behavior in the material, because the number of them is reduced to a few of tens or hundreds of units, this phenomenon is called quantum confinement. In case of luminescent properties, some QD semiconductors have been studied for optoelectronic applications due to the possibility for tuning the down conversion light emission in the photoluminescent process. Some precursor materials with which QDs are manufactured must own a wide electronic density, such some transition metals like $\mathrm{Cd}, \mathrm{Pb}, \mathrm{Zn}$ and $\mathrm{Se}, \mathrm{Te}, \mathrm{S}$ [3], therefore, except for $\mathrm{Zn}$, special care must be taken working on these, and sometimes take long time and difficult routes to obtain the desired material, particle size, crystallographic phase, etc.

In this work, we present a direct, facile and environmentally friendly route to obtain low toxicity high luminescent ZnS QDs, it consists of a direct mixture of 1:1 molar precursor solutions method. First, a solution was prepared by dissolving zinc nitrate $\left(\mathrm{Zn}\left(\mathrm{NO}_{3}\right)_{2} \cdot 6 \mathrm{H}_{2} \mathrm{O}\right)$ in deionized water in constant magnetic stirring for $15 \mathrm{~min}$. A second solution was prepared by dissolving sodium sulfide $\left(\mathrm{Na}_{2} \mathrm{~S} \cdot 9 \mathrm{H}_{2} \mathrm{O}\right)$ in deionized water with same molar concentration than the first solution. After 15 min stirring the second solution, we mixed both solutions and left to react for another $15 \mathrm{~min}$ at room temperature. The product was collected by centrifugation and dried to obtain a powder material for further characterization.

Morphological characterization of powder material was obtained by scanning electron microscopy (SEM and STEM mode), fig. 1a) show that we have very small particles forming the material, about 10 $\mathrm{nm}$ diameter but is not possible to know clearly. Figure 1b) shows a better STEM image that confirm a spherical particle with smaller size than $5 \mathrm{~nm}$. These results indicate that material is into confinement regime. Energy-dispersive X-ray Spectroscopy (EDS) indicates that a pure material was obtained, shown in figure 1c).

A micro-Raman spectroscopy study was carried out to determine the crystallographic structure of the material, in figure 2a) it is shown a known $\mathrm{ZnS}$ hexagonal wurtzite type phase Raman spectrum. The fundamental modes of that phase are located at 258, 307 and $343 \mathrm{~cm}^{-1}$ ( $\mathrm{E}_{1}(\mathrm{TO}), \mathrm{E}_{2}^{2}$ and $\mathrm{E}_{1}(\mathrm{LO})$, respectively)[4], all peaks show widening and shifting due to small particle sized material, in agreement with the SEM images. Photoluminescence (PL) study was made in the as prepared solution and over the powder material, both exhibiting the same behaviour. Fig. 2b) shows an intense absorbance band of $\mathrm{ZnS}$ 
QDs aqueous solution in UV region between $260 \mathrm{~nm}$ and $320 \mathrm{~nm}$, this due to an increased band gap value, also PL spectrum shows a high intensity blue light emission centred at 420-440 nm associated with 4 types of trap levels whose energy are located in the wide band gap of the material. Fig. 2c) shows the chromaticity location in CIE 1931 diagram. Usually, bulk ZnS presents a greenish emission spectrum centered around $500 \mathrm{~nm}$ because its band gap value[5], but in the current study, quantum confinement causes the observed blue shift.

Finally, we can conclude that ZnS QDs have very good properties that make it a potential component for the development of applications focused in a better use of light resources via downshifting (converting the UV light to visible region) for solar cell efficiency improvement applications and common white lighting (AlGaN UV based LEDs, for example) devices.

[1] N V Bondar and M S Brodyn, Physica E: Low-dimensional Systems and Nanostructures 42 (2010), p. 1549.

[2] S D Hodgson et al., Materials Letters 130 (2014), p. 120.

[3] Y-Q Liu et al., Optical Materials Express 7 (2017), p. 2161.

[4] O Brafman and S S Mitra, Physical Review 171 (1968), p. 931.

[5] D Van Thai et al., Optical and Quantum Electronics 48 (2016), p. 1.

[6] The authors acknowledge funding from Consejo Nacional de Ciencia y Tecnología (CONACyT)

México, through Project 255791-INFR 2015.
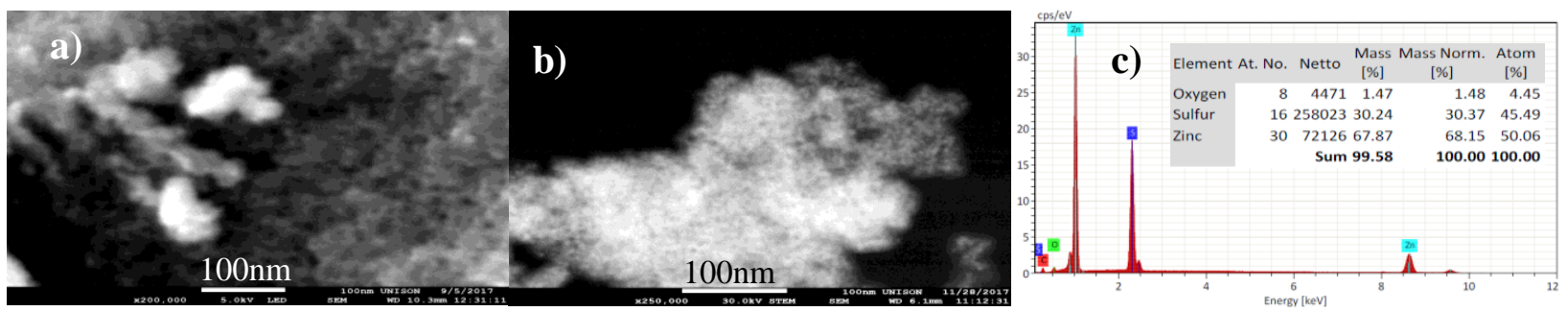

Figure 1. SEM image a) shows spherically shaped particles about 10nm of diameter, a better STEM image b) shows a smaller sized material around 5nm and EDS spectrum c) shows the composition of the material.
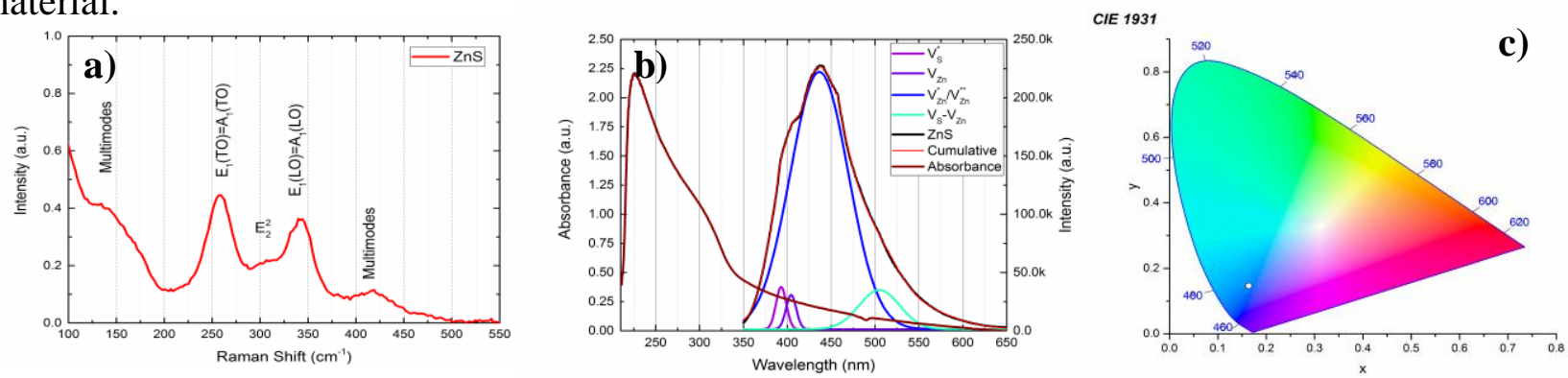

Figure 2. a) Raman spectrum shows the fundamental vibrational modes of hexagonal wurtzite type $\mathrm{ZnS}, \mathrm{b})$ absorbance and PL emission intensity $\left(\lambda_{\mathrm{ex}}=300 \mathrm{~nm}\right)$ at the left and right ordinate axis, respectively, of same ZnS QDs and c) shows the location of emission in CIE 1931 chromaticity diagram. 